\title{
The One-Dimensional KPZ Equation: an Exact Solution and its Universality
}

\author{
Tomohiro Sasamoto \\ Department of Mathematics and Informatics, Chiba University, 1-33 Yayoi-cho, Inage, Chiba 263-8522, Japar ${ }^{*}$ \\ Herbert Spohn \\ Zentrum Mathematik and Physik Department, TU München, D-85747 Garching, Germany
}

(Dated: today)

\begin{abstract}
We report on the first exact solution of the KPZ equation in one dimension, with an initial condition which physically corresponds to the motion of a macroscopically curved height profile. The solution provides a determinantal formula for the probability distribution function of the height $h(x, t)$ for all $t>0$. In particular, we show that for large $t$, on the scale $t^{1 / 3}$, the statistics is given by the Tracy-Widom distribution, known already from the theory of GUE random matrices. Our solution confirms that the KPZ equation describes the interface motion in the regime of weak driving force. Within this regime the KPZ equation details how the long time asymptotics is approached.
\end{abstract}

The motion of interfaces persists as a fascinating topic of statistical mechanics. One particular, intensely studied case are nonequilibrium growth processes, which are governed by local rules. In the seminal work 1 Kardar, Parisi, and Zhang proposed a model equation, now called KPZ equation, to investigate the dynamic scaling of such growing interfaces. KPZ argued that a growing interface has a statistically self-similar structure with universal scaling exponents for the interface width and for the transverse correlation length. In particular, they predicted that a one-dimensional interface has fluctuations which grow as $t^{1 / 3}$, in contrast to the $t^{1 / 4}$ statistical broadening of an equilibrium interface. Details of the KPZ scenario have been investigated through Monte Carlo simulations of simplified stochastic growth models, like Eden growth, random deposition, and polynuclear growth. Universal scaling exponents were confirmed, supporting that all such growth models constitute the $\mathrm{KPZ}$ universality class. On the theoretical side a variety of techniques have been devised, useful also in other areas of nonequilibrium statistical mechanics, as dynamic and functional renormalization group, mode-coupling theory, exact solutions, and more. For the development up to 1995 we refer to the book 2 and the reviews 3, 4. However, so far the KPZ equation itself has resisted analytical handling. We will report here on the first exact solution. Physically it describes cluster growth in a thin film. The solution also provides a better understanding of the universality of the KPZ equation.

We will be concerned with the one-dimensional KPZ equation only. The interface location is then described by a height function $h(x, t)$ depending on time $t$ and location $x$ on the real line. The KPZ equation reads

$$
\frac{\partial}{\partial t} h=\frac{1}{2} \lambda\left(\frac{\partial}{\partial x} h\right)^{2}+\nu \frac{\partial^{2}}{\partial x^{2}} h+\sqrt{D} \eta .
$$

The first term is the slope dependent growth velocity of strength $\lambda, \lambda>0$ for convenience. The Laplacian smoothens the interface with diffusivity $\nu>0$. $\eta$ is normalized space-time white noise with correlator $\left\langle\eta(x, t) \eta\left(x^{\prime}, t^{\prime}\right)\right\rangle=\delta\left(x-x^{\prime}\right) \delta\left(t-t^{\prime}\right)$ and $\sqrt{D}$ is the noise strength. $\eta$ models the random nucleations at the interface. We will choose initial conditions such that the height profile remains curved on the macroscopic scale.

In the breakthrough contribution [5] Johansson firstly succeeded to compute a universal probability distribution function (pdf), see also the related work [6, 7]. He studied a discrete growth model, known as single step, with wedge initial profile. Most surprisingly, he discovered that the pdf for the random amplitude of the height is the Tracy-Widom distribution [8], first obtained in the context of the large $N$ statistics of the largest eigenvalue of a GUE random matrix 9. The Tracy-Widom pdf, $\rho_{\mathrm{TW}}(s)$, is defined through the determinant of a symmetric operator acting on functions on the real line, where, in principle, the determinant is defined by the product of the eigenvalues. More explicitly, we denote by $P_{s}$ the projection operator onto the interval $[s, \infty)$ and by $K_{\mathrm{Ai}}$ the Airy kernel

$$
K_{\mathrm{Ai}}(x, y)=\int_{0}^{\infty} \mathrm{d} w \operatorname{Ai}(x+w) \operatorname{Ai}(y+w)
$$

with Ai the standard Airy function. Then

$$
\rho_{\mathrm{TW}}(s)=\frac{d}{d s} \operatorname{det}\left(1-P_{s} K_{\mathrm{Ai}} P_{s}\right) .
$$

One can show that $\operatorname{tr}\left[P_{s} K_{\mathrm{Ai}} P_{s}\right]<\infty$ [8] and hence the infinite product makes sense.

In a spectacular recent experiment on electroconvection 10 the Tracy-Widom statistics is verified down to a scale of height samples with probability $10^{-4}$. In this experiment a thin film of liquid crystal is electrically driven to a turbulent phase, the unstable DSM1 phase. One then plants through a laser pulse a sharply localized seed of topological-defect turbulence, the stable DSM2 phase. It grows isotropically over a time window of $30 \mathrm{sec}$ to the maximal size of $1.6 \mathrm{~cm}$ in diameter. The experiment also investigates how the Tracy-Widom distribution is approached for long times. One observes that the cumulants 2, 3, and 4 have already reached their Tracy-Widom 
(a)



(b)

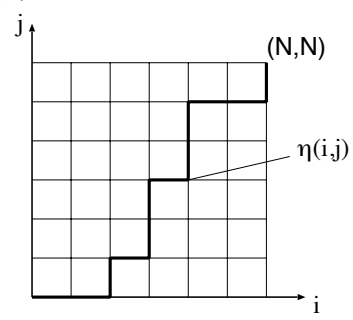

FIG. 1. (a) A typical realization of the droplet height function. (b) A directed polymer configuration.

value, while the average still slowly decays as $t^{-1 / 3}$ towards its stationary value, an observation so far without theoretical explanation.

Exact solution.-The curved height profile can locally be approximated by a parabola. In idealization, we therefore choose our initial conditions such that the average height is exactly parabolic, which can be achieved through the initial sharp wedge

$$
h(x, 0)=-|x| / \delta, \quad \delta \ll 1 .
$$

Since for short times the nonlinear term dominates, the solution $h(x, t)$ spreads rapidly into the parabolic profile, $h(x, t) \simeq-x^{2} / 2 \lambda t$ for $|x| \leq \lambda t / \delta$ and $h(x, t) \simeq\left(\lambda / 2 \delta^{2}\right) t-$ $|x| / \delta$ for $|x| \geq \lambda t / \delta$. Because of the noise term in (1) this profile has superimposed random fluctuations whose amplitude grows as $t^{1 / 3}$. A typical realization is shown in Fig. 1(a).

We consider the height statistics at one prescribed point $x$. Then, for every $t>0$,

$$
(\lambda / 2 \nu) h(x, t)=-x^{2} / 4 \nu t-\frac{1}{12} \gamma_{t}^{3}+2 \log \alpha+\gamma_{t} \xi_{t},
$$

where

$$
\gamma_{t}=\left(\alpha^{4} \nu t\right)^{1 / 3}, \quad \alpha=(2 \nu)^{-3 / 2} \lambda D^{1 / 2} .
$$

The first three terms of (5) are deterministic, in particular one notes the inverted parabola already mentioned. The logarithmic term reflects the scale invariance of the initial sharp wedge. All information on the fluctuations of $h(x, t)$ is encoded in the random amplitude $\xi_{t}$, which extends the Tracy-Widom amplitude, $\xi_{\mathrm{TW}}$, to finite time $t$. The prefactor $\gamma_{t}$ confirms that the fluctuations grow as $t^{1 / 3}$. The pdf of $\xi_{t}$ is given by

$$
\begin{aligned}
& \rho_{t}(s)=\int_{-\infty}^{\infty} \mathrm{d} u \gamma_{t} \mathrm{e}^{\gamma_{t}(s-u)} \exp \left[-\mathrm{e}^{\gamma_{t}(s-u)}\right] \\
& \times\left(\operatorname{det}\left(1-P_{u}\left(B_{t}-P_{\mathrm{Ai}}\right) P_{u}\right)-\operatorname{det}\left(1-P_{u} B_{t} P_{u}\right)\right) .
\end{aligned}
$$

Here $P_{\mathrm{Ai}}$ has the integral kernel $\operatorname{Ai}(x) \operatorname{Ai}(y)$, which can be viewed as a one-dimensional unnormalized projection, and $B_{t}$ has the kernel

$$
B_{t}(x, y)=\int_{-\infty}^{\infty} \mathrm{d} w\left(1-\mathrm{e}^{-\gamma_{t} w}\right)^{-1} \operatorname{Ai}(x+w) \operatorname{Ai}(y+w) .
$$

$\xi_{t}$ is independent of $x$ and depends on $t$ only through the dimensionless parameter $\gamma_{t}$. In the limit $t \rightarrow \infty$ the Gumbel density in (7) tends to $\delta(s-u)$ and $B_{t}$ tends to $B_{\infty}=K_{\mathrm{Ai}}$. The difference of determinants in (7) is then precisely $\rho_{\mathrm{TW}}(s)$. Hence $\xi_{t}$ tends to $\xi_{\mathrm{TW}}$ as $t \rightarrow \infty$. In case of a macroscopically curved profile, it is expected that for any growth process in the KPZ class the pdf of the height fluctuations is Tracy-Widom in the long time limit. We have thus established that the KPZ equation is in the KPZ universality class as regards to the pdf of the height fluctuations.

Our exact solution also provides an explanation for the observed $t^{-1 / 3}$ relaxation to stationarity. The difference of determinants in (7) is of order $t^{-4 / 3}$ [11. Hence the leading correction to $\rho_{\mathrm{TW}}(s)$ is the shift due to the nonzero mean of the Gumbel distribution which is of order $t^{-1 / 3}$.

To represent the solution to (11) in a more tractable form we introduce the Cole-Hopf transformation as

$$
Z(x, t)=\exp [(\lambda / 2 \nu) h(x, t)] .
$$

At the expense of multiplicative noise, $Z(x, t)$ then satisfies the linear equation

$$
\frac{\partial}{\partial t} Z=\nu \frac{\partial^{2}}{\partial x^{2}} Z+(\lambda \sqrt{D} / 2 \nu) \eta Z .
$$

with the normalized initial condition $Z(x, 0)=$ $\lim _{\delta \rightarrow 0}((2 \nu / \lambda) 2 \delta)^{-1} \exp [-(\lambda / 2 \nu)|x| / \delta]=\delta(x)$. 10 is solved through the Feynman path integral

$$
Z(x, t)=\mathbb{E}_{0}\left(\exp \left[\alpha \int_{0}^{2 \nu t} \mathrm{~d} s \eta(b(s), s)\right] \delta(b(2 \nu t)-x)\right) .
$$

Here $\mathbb{E}_{0}(\cdot)$ is the Wiener integral over all paths of an auxiliary Brownian motion $b(t)$, starting at 0 and with variance $t$. In principle, the solution of the KPZ equation with sharp wedge initial condition is defined by

$$
h(x, t)=(2 \nu / \lambda) \log Z(x, t) .
$$

However, as written $\langle Z(x, t)\rangle=\infty$ because of ultraviolet divergencies. As in quantum field theory one thus has to introduce a suitable cutoff to be removed through a renormalization scheme. We briefly describe four distinct variants, all providing physical insight to the interpretation of the KPZ equation. But only the last variant carries the computational power to obtain the exact solution.

(i) Colored noise.-The expression (11) is only formal, since the white noise $\eta$ is very rough and hence the action in (11) relies on an undefined integral. One way to improve the situation is to substitute in the KPZ equation (1) the noise $\eta(x, t)$ by a noise $\eta_{\kappa}(x, t)$, which is colored in $x$ with width $\kappa^{-1}$ but still white in $t$, such that $\eta_{\kappa} \rightarrow \eta$ as $\kappa \rightarrow \infty$. This modification induces the uniform translation of the height profile by $v_{\kappa} t$ with $v_{\kappa} \simeq \kappa$. Going 
to the moving frame of reference, the limit $\kappa \rightarrow \infty$ of the height profile is well defined [12, 13] and yields the proper interpretation of (11).

(ii) Directed polymer in a random potential.-The integral in (11) is discretized. Then the Brownian motion path $b(t)$ is replaced by a directed polymer $\omega$, which for convenience is placed on the two-dimensional lattice $\mathbb{Z}^{2}$. It starts at 0 , makes only up/right moves, and ends at the lattice site $(t N, t N)$, see Fig. 1(b). Independently for each site $(i, j) \in \mathbb{Z}^{2}$, there is a unit Gaussian random potential $\eta(i, j)$. The energy of the directed polymer is $E(\omega)=\sum \eta(i, j)$, where the sum is over the potentials along the polymer $\omega$. The discrete approximation to (11) reads then

$$
Z_{t N}=\sum_{\omega:(0,0) \sim(t N, t N)} \exp [-\beta E(\omega)] .
$$

The partition function is point-to-point, since both endpoints of the polymer are fixed. In the limit $N \rightarrow \infty$, $\beta \rightarrow 0$ with $\beta^{4} N$ fixed, $Z_{t N} \rightarrow Z(0, t)$ and $Z(0, t)$ as defined in (i) 14. From this perspective the KPZ equation is the weak noise limit of the directed polymer, see [15] for details.

(iii) Attractive $\delta$-Bose gas [16].-The $n$-th moment, $\left\langle Z(x, t)^{n}\right\rangle$, of the partition function (11) can be expressed through the propagator of $n$ quantum particles on the line interacting through an attractive $\delta$-potential. All $n$ particles start at 0 and propagate to $x$ at time $t$. Working out $\left\langle Z^{n}\right\rangle$ yields the potential $-\frac{1}{2} \alpha^{2} \sum_{i, j=1}^{n} \delta\left(x_{i}-x_{j}\right)$. Thus the renormalization corresponds to merely removing the self-energy through normal ordering. Thereby one arrives at the same random partition function as constructed in (i) and (ii).

(iv) Single step growth model.-This is a stochastic evolution model for the integer valued height function $h(j, t), j \in \mathbb{Z}$, satisfying the single step constraint

$$
|h(j+1, t)-h(j, t)|=1 .
$$

In our case the initial height profile is the wedge $h(j, 0)=$ $-|j|$. A random sequential update rule is used: Independently the height at a local minimum is increased by 2 with rate $p$ and at a local maximum decreased by 2 with rate $q, p+q=1$ to set the time scale, and $q>p$ in our case corresponding to $\lambda>0$. The height differences are then governed by the partially asymmetric simple exclusion process (PASEP). This is a stochastic particle system on $\mathbb{Z}$, where there is at most one particle per site. Particles jump with rate $p$ to the right and rate $q$ to the left under the constraint of the exclusion rule. The initial wedge corresponds to the $0-1$ step initial particle configuration, for which all sites to left of the origin are empty and to the right of the origin are filled. In [5] the TracyWidom fluctuations are proved in case $p=0, q=1$ (the TASEP), including a discrete time parallel random update rule. The intricate extension to $0<p<q<1$ has been accomplished by Tracy and Widom [17, 18]. Also available is the corresponding result for the PNG droplet [6, 19, 20.

The Cole-Hopf solution (11) arises at the crossover scale to weak asymmetry (WASEP). More specifically, one assumes $q=\frac{1}{2}+\sqrt{\varepsilon} \beta, \beta>0, \varepsilon>0$, and $\varepsilon \ll 1$. The correspondingly adjusted time scale is order $\varepsilon^{-2}$ and space scale is order $\varepsilon^{-1}$, while the height is of order $\varepsilon^{-1 / 2}$. If $h^{\varepsilon}(j, t)$ denotes the WASEP random height profile, then the single step partition function is defined through

$$
Z_{\text {step }}^{\varepsilon}(j, t)=\exp \left[(\log (p / q)) h^{\varepsilon}(j, t)\right] .
$$

As shown in [12, 13, the correctly centered $Z_{\text {step }}^{\varepsilon}\left(\varepsilon^{-1} x, \varepsilon^{-2} t\right)$ converges in the limit $\varepsilon \rightarrow 0$ to $Z(x, t)$, constructed already before in (i) - (iii).

We are now in a position to indicate, rather roughly, how our exact solution is obtained. A more detailed exposition can be found in [11, 21. For the $0-1$ step initial particle configuration, Tracy and Widom [17 recently provided a contour integration formula for the probability distribution of the position of the $m$-th particle at time $t$ valid for arbitrary $q$. In 21] we use their formula as starting point and study the scaling limit of the PASEP as above, namely asymmetry $\sqrt{\varepsilon} \beta$, space $\mathcal{O}\left(\varepsilon^{-1}\right)$, and time $\mathcal{O}\left(\varepsilon^{-2}\right)$, to arrive at a pdf given as an integral over the difference of two determinants as in (7), including a deterministic shift of order $\varepsilon^{-1}$ and a subleading $\log \varepsilon$ correction. As discussed in the companion paper [11, in addition one has to analyze the average $\left\langle Z_{\text {step }}^{\varepsilon}(j, t)\right\rangle$ for the WASEP, thereby to determine the appropriate centering. One finds terms of order $\varepsilon^{-1}$ and $\log \varepsilon$, which are precisely cancelled by the corresponding terms appearing in the WASEP scaling limit. Combining both results, one arrives at (5) - 8). The KPZ parameters are fixed through the WASEP as $2 \nu=1$ and $\beta=\lambda$. At $x=0$ the WASEP average density is $1 / 2$ and hence the noise strength $D=1 / 4$. By varying $x$ one can tune the noise strength to $\rho(1-\rho)$, where $\rho$ is the local average density. In principle it suffices to consider $x=0$ and to deduce the parameter dependence from (11).

For a more detailed information on the pdf $\rho_{t}(s)$ one has to rely on a numerical evaluation of (7), for which purpose it is convenient to regard, e.g., $P_{u} B_{t} P_{u}$ as a large matrix. One then evaluates $B_{t}(x, y)$ at a judiciously chosen set of grid points $x_{j}, j=1, \ldots, n, u \leq x_{j}$, with $n$ of order 60 to 120 and thereby defines the $n \times n$ matrix $B_{i j}=B_{t}\left(x_{i}, x_{j}\right), i, j=1, \ldots, n$, for which $\operatorname{det}(1-B)$ is obtained by a standard routine. Since the kernel of $(8)$ is a smooth function, the computation is fast and the errors are small [22]. In Fig. 2(a) we display the pdf $\rho_{t}(s)$ for $\gamma_{t}=2,5,10$ obtained by the method described. For even smaller values of $\gamma_{t}$ the computation becomes slow because of strong oscillations of the kernel $B_{t}(x, y)$ resulting from the singularity at $w=0$ of the defining integral (8). 


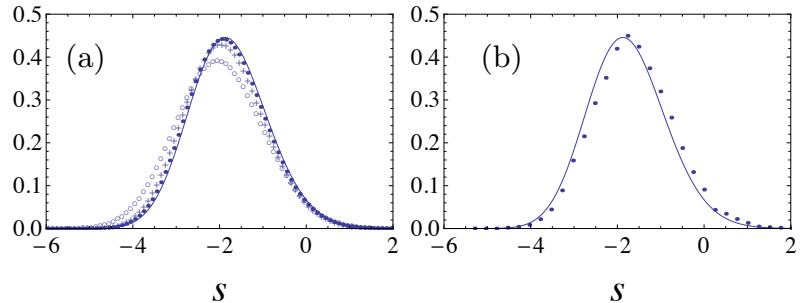

FIG. 2. (a) The pdf defined by (7) for $\gamma_{t}=2(-), 5(+), 10(\cdot)$ and the Tracy-Widom pdf defined by (3) (full curve). (b) The TASEP height statistics at $10^{3}$ MC steps and the TracyWidom pdf.

Universal properties. - The exact solution has been obtained through a limit of vanishing asymmetry. More generally, one could consider some growth model in the KPZ class with a tunable asymmetry, denoted by $\beta$. Physically the asymmetry is possibly small, but fixed. Time is measured in Monte Carlo time steps. For times of order $\beta^{-2}$ the nonlinearity plays no role, yet, and the height fluctuations are approximately Gaussian. On the time scale $\beta^{-4}$, one crosses over to the KPZ solution (5) with non-Gaussian statistics, which asymptotically is well approximated by the Tracy-Widom distribution. Of course, a clean separation of time scales is expected only for $\beta \ll 1$.

To have a test case we performed MC simulations for the PASEP with strongest possible asymmetry, namely $q=1, p=0$ (the TASEP). For the $0-1$ step initial condition we sampled the height at the origin, $h_{q=1}(0, t)$. The scale linear in $t$ is given by $-(q-p) t / 2$. For $t=10^{3}$ MC steps the distribution of $h_{q=1}(0, t)$ is concentrated approximately in an interval of 60 lattice units. As displayed in Fig. 2(b), even on the discrete level the TracyWidom distribution is still an accurate approximation, which becomes almost indistinguishable upon shifting it by 0.13 units to the right. The $t^{-1 / 3}$ shift to the right was noted before numerically from the recursion relations of the PNG droplet 23 and is also observed in the experiment [10]. According to the exact solution, for large $t, \rho_{t}$ is shifted by $\gamma_{t}^{-1}(-0.577+2 \log \alpha)$ relative to $\rho_{\mathrm{TW}}$, where -0.577 is the mean of the Gumbel density. Since $\alpha$ is proportional to $\lambda$, this suggests that the prefactor of the $t^{-1 / 3}$ shift for the PASEP should undergo a sign change. Indeed, running the MC simulation for smaller values of $q$, one finds that $\rho_{t}$ is shifted to the left side of $\rho_{\mathrm{TW}}$ for $q \lesssim 0.78$. The approach as $t^{-1 / 3}$ seems to be universal and is expected to hold for any growth model in the KPZ class. However the precise first order correction to $\rho_{\mathrm{TW}}(s)$ will depend on the particular model.

Conclusions. - We have computed the exact probability distribution function for the height $h(x, t)$ of the KPZ equation with narrow wedge initial profile. The long time limit is the Tracy-Widom pdf, in accordance with the results for discrete growth models. But in addition, based on our exact solution, we now better understand how the long time asymptotic is approached. For growth models with tunable asymmetry the KPZ equation is an accurate approximation for weak asymmetry.

Note.-After submitting our letter, three distinct groups [13, 15, 24] posted independent but related work on the arXiv.

We are grateful to Michael Prähofer for many illuminating discussions. H. S. thanks Jeremy Quastel for emphasizing the importance of the crossover WASEP. This work is supported by a DFG grant. In addition T.S. acknowledges the support from KAKENHI (9740044) and H.S. from Math-for-Industry of Kyushu University.

* sasamoto@math.s.chiba-u.ac.jp

† spohn@ma.tum.de

[1] M. Kardar, G. Parisi, Y-C. Zhang, Phys. Rev. Lett. 56, 889-892 (1986).

[2] A.-L. Barabási, H.E. Stanley, Fractal Concepts in Surface Growth, Cambridge University Press, 1995.

[3] T. Halpin-Healy, Y-C. Zhang, Phys. Rep. 254, 215-415 (1995).

[4] J. Krug, Advances in Physics 46, 139-282 (1997).

[5] K. Johansson, Comm. Math. Phys. 209, 437-476 (2000).

[6] J. Baik, P. Deift, K. Johansson, J. Amer. Math. Soc. 12, 1119-1178 (1999).

[7] M. Prähofer, H. Spohn, Phys. Rev. Lett. 84, 4882-4885 (2000)

[8] C.A. Tracy, H. Widom, Comm. Math. Phys. 159, 151174 (1994).

[9] P.J. Forrester, Nucl. Phys. B 402, 709-728 (1993).

[10] K.A. Takeuchi, M. Sano, arXiv:1001.5121, preprint (2010).

[11] T. Sasamoto, H. Spohn, arXiv:1002.1879, Nuclear Phys. B, online (2010).

[12] L. Bertini, G. Giacomin, Comm. Math. Phys. 183, 571607 (1997).

[13] G. Amir, I. Corwin, J. Quastel, arXiv:1003.0443, preprint (2010).

[14] T. Alberts, K. Khanin, J. Quastel, arXiv:1003.1885 preprint (2010).

[15] P. Calabrese, P. Le Doussal, A. Rosso, arXiv:1002.4560 Eur. Phys. Lett., to appear (2010).

[16] M. Kardar, Statistical Mechanics of Fields, Cambridge University Press, 2007.

[17] C.A. Tracy, H. Widom, Comm. Math. Phys. 290, 129154 (2009).

[18] C.A. Tracy, H. Widom, J. Math. Phys. 50, 095204 (2009).

[19] M. Prähofer, H. Spohn, J. Stat. Phys. 108, 1071-1106 (2002).

[20] A. Borodin, P.L. Ferrari, M. Prähofer, T. Sasamoto, J. Stat. Phys. 129, 1055-1080 (2007).

[21] T. Sasamoto, H. Spohn, arXiv:1002.1873, J. Stat. Phys., to be published, (2010).

[22] F. Bornemann, Math. Comp., online (2009).

[23] M. Prähofer, private communication, 2009.

[24] V. Dotsenko, arXiv:1003.4899, Eur. Phys. Lett., to appear (2010). 